\title{
Scanning Electron Microscopy as a New Tool for Diagnostics in Pathology
}

\author{
Tzipi Cohen Hyams ${ }^{1 *}$ and Murray C. Killingsworth ${ }^{2-4}$
}

1. Ingham Institute for Applied Medical Research, Liverpool, NSW, Australia.

2. Department of Anatomical Pathology, New South Wales Health Pathology, Liverpool, NSW, Australia.

3. Faculty of Medicine, University of New South Wales, Sydney, NSW, Australia.

4. School of Medicine, University of Western Sydney, Sydney, NSW, Australia.

* Corresponding author: tzipi.cohen-hyams@inghaminstitute.org.au

Pathologists generally examine micrometer-thin tissue slices by means of brightfield light microscopy (LM) and transmission electron microscopy (TEM) [1] in order to identify cellular changes and diagnose disease. Scanning electron microscopy (SEM) is generally believed to be non-contributory to ultrastructural studies of disease as early SEM studies were mainly used to image sample topography [2] rather than the cell interior. In this paper, we present an alternative to TEM with the new generation high-resolution SEMs (HRSEM) that not only have equivalent performance but exhibit new capabilities and applications that can be usefully employed for diagnostic pathology and cell biology. HRSEM has important and crucial advantages over TEM. It is not limited by sample thickness ( $\sim 100 \mathrm{~nm}$ thick) or by beam damage to delicate structures, such as cytoskeletal filaments. HRSEM allows manual and automated re-imaging as many times as needed with different electron signals. Additionally, the cost of HRSEM, its operation and its maintenance are considerably lower than for TEM. Current high-end HRSEMs have automated scan generation systems such as the new integrated system ATLAS 5 from Carl Zeiss, Germany [3] and Maps from ThermoFisher, USA [4]. Lastly, it is now possible to view both cell internal structure in STEM mode and external macromolecular structures in two and three dimensions, thereby enhancing depth information lacking in conventional microscopic studies.

Prostate tissues from a human patient and renal tissues from an animal model were routinely processed as for TEM imaging [5]. 120-250 nm thick epoxy resin sections were cut with an ultramicrotome (RMC Boeckeler, USA) and mounted either on carbon coated glass histological slides, silicon wafer or 300 mesh copper TEM grids. The samples were carbon coated using a SEM carbon coater (Agar Scientific, UK). Imaging was performed using the Angular selective backscattered detector (AsB) and the Atlas 5.2 in tile scan mode attached to the GeminiSEM 300 (Carl Zeiss, Germany) equipped with field emission gun at $7 \mathrm{kV}$, at a working distance of $3.5 \mathrm{~mm}$ approximately and also using STEM mode at $30 \mathrm{kV}$ and at a working distance of $2.8 \mathrm{~mm}$ as shown in Figures 1-2.

While the ultrastructural examination of human biopsies can be of great value in general pathology, it is in renal pathology that electron microscopy is considered to be an essential component. The HRSEM micrographs present clearly the ultrastructural features of the normal glomerular tuft within Bowman's capsule (Figure 1a, 1b). The SEM micrographs present a normal glomerular capillary loop and mesangial region in similar detail to that of a TEM micrograph [6]. It is clear that the mesangial matrix is continuous with the basement membrane and separate to the mesangial cell cytoplasm.

HRSEM systems also facilitate wide-area survey views of complex tissue. Figures $2 \mathrm{a}$ and $2 \mathrm{~b}$ present images from a human radical prostatectomy sample focusing on the larger veins growing in close proximity to the edge of an adenocarcinoma. The growing endothelial sprouts emanating from these 
large veins could effectively bring the vasculature into closer contact with the tumor cells. These sprouts appear to be more numerous on aspects of the vein closer to the tumour front. This finding has stimulated future research to assess the role of anti-angiogenic therapy as an effective strategy for reducing tumor metastasis. It highlights the possibility that angiogenesis may facilitate the entry of breakaway tumor cells into the vasculature wherein they become circulating tumor cells capable of initiating tumor metastatic spread.

We believe that HRSEM will follow a similar evolutionary course to TEM in the field of diagnostic pathology and that this technology will ultimately be established as an essential diagnostic tool.

\section{References:}

[1] KE Carr and PG Toner, Journal of Microscopy 123 (1981), p. 147.

[2] A Bogner et al., Micron, 38 (2007), p. 390.

[3] Zeiss, https://www.zeiss.com/microscopy/int/products/microscope-software/atlas.html (current).

[4] Thermo Fisher, https://www.fei.com/software/maps/ (current).

[5] MC Killingsworth and X Wu, Pathobiology 78 (2011), p. 24.

[6] J Moss and I Shore, Pathology 8 (2002), p. 207.

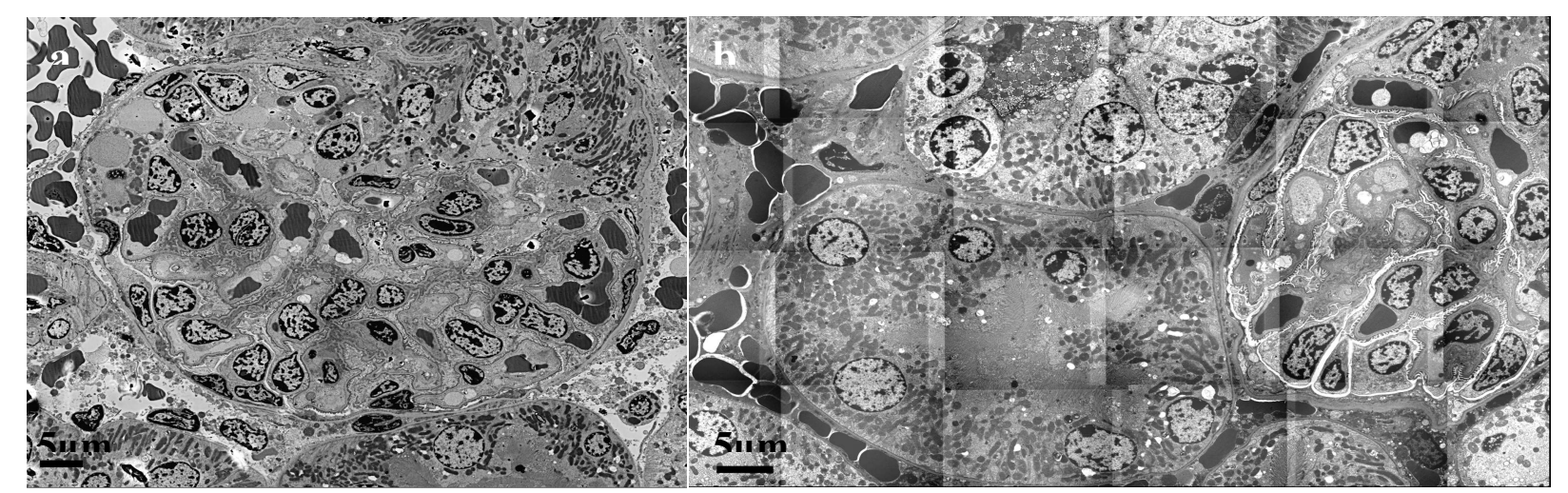

Figure 1. a-b SEM and STEM micrographs of a renal mouse tissue, respectively demonstrating the ultrastructural features of the normal glomerular tuft within Bowman's capsule.
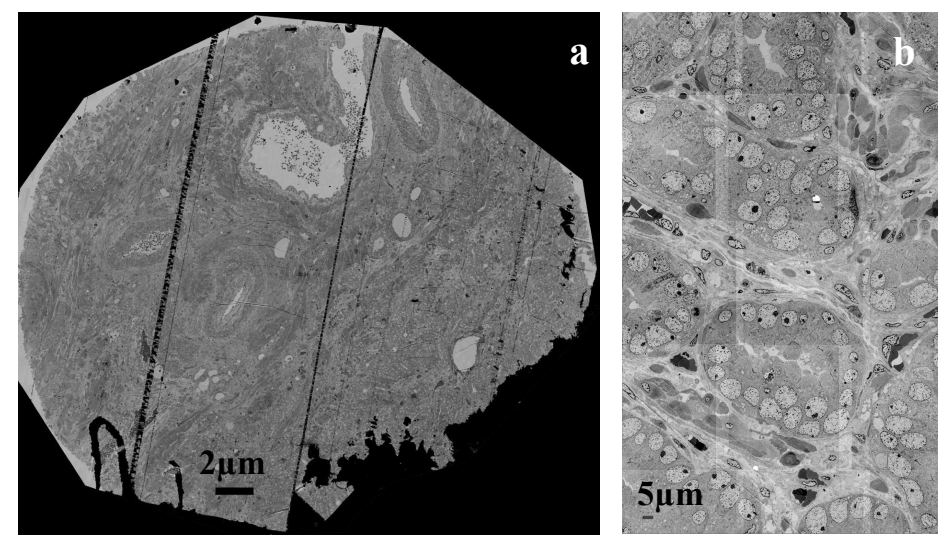

Figure 2. a-b SEM and STEM micrographs of a prostate human tissue, respectively showing close proximity of the tumour front to the vasculature. 